\title{
A well of loneliness : diversity among leading Linux kernel developers (2005-2020)
}

\author{
Camille Akmut
}

February 5, 2020

\begin{abstract}
Fifteen years of Linux kernel development are reviewed from the point of view of gender diversity. Study based on total commits (as opposed to e.g. mailing list participation) $: \geq 1,400$, corresponding to top 50 Linux kernel developers (including Linus Torvalds).
\end{abstract}


Table 1 - Diversity among Linux kernel developers (2005-2020)

\begin{tabular}{|c|c|c|c|c|}
\hline & & commits & & \\
\hline 1 & tiwai & 6,207 & Takashi Iwai & $\mathrm{m}$ \\
\hline 2 & ickle & 5,401 & $?$ & $?$ \\
\hline 3 & arndb & 4,633 & Arnd Bergmann (?) & $\mathrm{m}(?)$ \\
\hline 4 & jmberg & 4,435 & Johannes Berg (?) & $\mathrm{m}(?)$ \\
\hline 5 & gregkh & 4,344 & Greg Kroah-Hartman & $\mathrm{m}$ \\
\hline 6 & bigguiness & 4,196 & H Hartley Sweeten & $\mathrm{m}$ \\
\hline 7 & htejun & 4,132 & Tejun Heo & $\mathrm{m}$ \\
\hline 8 & broonie & 4,060 & Mark Brown & $\mathrm{m}$ \\
\hline 9 & mchehab & 3,988 & Mauro Carvalho Chehab & $\mathrm{m}$ \\
\hline 10 & morimoto & 3,387 & Kuninori Morimoto & $\mathrm{m}$ \\
\hline 11 & davem330 & 3,217 & David S. Miller & $\mathrm{m}$ \\
\hline 12 & danvet & 3,187 & Daniel Vetter & $\mathrm{m}$ \\
\hline 13 & JoePerches & 3,131 & Joe Perches (?) & $\mathrm{m}(?)$ \\
\hline 14 & skeggsb & 3,115 & Ben Skeggs (?) & $\mathrm{m}(?)$ \\
\hline 15 & vsyrjala & 3,102 & Ville Syrjala (?) & $\mathrm{m}(?)$ \\
\hline 16 & linusw & 2,971 & Linus Walleij & $\mathrm{m}$ \\
\hline 17 & AxelLin & 2,929 & Axel Lin & $\mathrm{m}$ \\
\hline 18 & ColinIanKing & 2,676 & Colin Ian King & $\mathrm{m}$ \\
\hline 19 & dhowells & 2,452 & David Howells (?) & $\mathrm{m}(?)$ \\
\hline 20 & bzolnier & 2,424 & Bartlomiej Zolnierkiewicz (?) & $\mathrm{m}(?)$ \\
\hline 21 & jwrdegoede & 2,316 & Hans de Goede & $\mathrm{m}$ \\
\hline 22 & larsclausen & 2,302 & Lars-Peter Clausen & $\mathrm{m}$ \\
\hline 23 & andy-shev & 2,288 & Andy Shevchenko & $\mathrm{m}$ \\
\hline 24 & jhovold & 2,146 & Johan Hovold & $\mathrm{m}$ \\
\hline 25 & ralfbaechle & 2,123 & Ralf Baechle & $\mathrm{m}$ \\
\hline 26 & masahir0y & 2,060 & Masahiro Yamada & $\mathrm{m}$ \\
\hline 27 & ebiederm & 1,934 & Eric Biederman (?) & $\mathrm{m}(?)$ \\
\hline 28 & AdrianBunk & 1,914 & Adrian Bunk & $\mathrm{m}$ \\
\hline 29 & paulmck & 1,847 & Paul E. McKenney & $\mathrm{m}$ \\
\hline 30 & heicarst & 1,819 & Heiko Carstens (?) & $\mathrm{m}(?)$ \\
\hline 31 & vireshk & 1,752 & Viresh Kumar & $\mathrm{m}$ \\
\hline 32 & herbertx & 1,732 & Herbert Xu & $\mathrm{m}$ \\
\hline 33 & ffainelli & 1,730 & Florian Fainelli & $\mathrm{m}$ \\
\hline 34 & groeck & 1,691 & Guenter Roeck & $\mathrm{m}$ \\
\hline 35 & hverkuil & 1,676 & Hans Verkuil (?) & $\mathrm{m}(?)$ \\
\hline 36 & tmlind & 1,656 & Tony Lindgren & $\mathrm{m}$ \\
\hline 37 & torvalds & 1,595 & Linus Torvalds & $\mathrm{m}$ \\
\hline 38 & kees & 1,575 & Kees Cook & $\mathrm{m}$ \\
\hline 39 & jankara & 1,554 & Jan Kara & $\mathrm{m}$ \\
\hline 40 & ozbenh & 1,543 & Benjamin Herrenschmidt & $\mathrm{m}$ \\
\hline 41 & chucklever & 1,507 & Chuck Lever & $\mathrm{m}$ \\
\hline
\end{tabular}




\begin{tabular}{|l|l|l|l|l|}
\hline 42 & neilbrown & 1,501 & Neil Brown & $\mathrm{m}$ \\
\hline 43 & mripard & 1,473 & Maxime Ripard & $\mathrm{m}$ \\
\hline 44 & mpe & 1,471 & Michael Ellerman & $\mathrm{m}$ \\
\hline 45 & pinchartl & 1,456 & Laurent Pinchart (?) & $\mathrm{m}(?)$ \\
\hline 46 & krzk & 1,456 & Krzysztof Kozlowski & $\mathrm{m}$ \\
\hline 47 & ian-abbott & 1,444 & Ian Abbott & $\mathrm{m}$ \\
\hline 48 & kaber & 1,427 & Patrick McHardy & $\mathrm{m}$ \\
\hline 49 & GustavoARSilva & 1,426 & Gustavo A. R. Silva & $\mathrm{m}$ \\
\hline 50 & djbw & 1,416 & Dan Williams & $\mathrm{m}$ \\
\hline
\end{tabular}

Table 1: "...to master, excluding merge commits" /torvalds/linux/

\section{Measured 05/02/20 c. 9.40 am (no refresh)}

Gender was identified based on names, photos, user names, 'Linux kernel report', mailing lists, commit messages...

An error would have consisted to give for time period "2002-2020" — otherwise indicated by Github. As the Linux kernel developer report makes additionally clear : the "git era" of Linux kernel development starts in $2005^{1}$ (git was released that year...).

\section{Table 2 - Aggregated results}

\begin{tabular}{|l|l|l|}
\hline Gender & $\mathrm{n}$ & Percentage \\
\hline Female & 0 & $0 \%$ \\
\hline Male & 50 & $100 \%$ \\
\hline
\end{tabular}

Table 2: Diversity among top 50 Linux kernel developers (2005-20)

\footnotetext{
1" the beginning of the git era (the 2.6.11 release in 2005)". Linux Foundation 2017 : 11.
} 
Table 3 - Diversity among Linux kernel developers (2005-2017)

\begin{tabular}{|l|l|l|}
\hline Name & "Changes" / \% & \\
\hline H Hartley Sweeten & $6,0340.9 \%$ & $\mathrm{~m}$ \\
\hline Al Viro & $5,9040.9 \%$ & $\mathrm{~m}$ \\
\hline Takashi Iwai & $5,0890.8 \%$ & $\mathrm{~m}$ \\
\hline Mauro Carvalho Chehab & $5,0390.8 \%$ & $\mathrm{~m}$ \\
\hline David S. Miller & $4,0440.6 \%$ & $\mathrm{~m}$ \\
\hline Johannes Berg & $4,0140.6 \%$ & $\mathrm{~m}$ \\
\hline Mark Brown & $3,9780.6 \%$ & $\mathrm{~m}$ \\
\hline Tejun Heo & $3,9510.6 \%$ & $\mathrm{~m}$ \\
\hline Russell King & $3,6920.6 \%$ & $\mathrm{~m}$ \\
\hline Greg Kroah-Hartman & $3,5930.6 \%$ & $\mathrm{~m}$ \\
\hline Thomas Gleixner & $3,5820.6 \%$ & $\mathrm{~m}$ \\
\hline Christoph Hellwig & $3,4980.5 \%$ & $\mathrm{~m}$ \\
\hline Hans Verkuil & $3,4190.5 \%$ & $\mathrm{~m}$ \\
\hline Ingo Molnár & $3,1280.5 \%$ & $\mathrm{~m}$ \\
\hline Chris Wilson & $3,0900.5 \%$ & $\mathrm{~m}$ \\
\hline Arnd Bergmann & $3,0710.5 \%$ & $\mathrm{~m}$ \\
\hline Geert Uytterhoeven & $3,0110.5 \%$ & $\mathrm{~m}$ \\
\hline Dan Carpenter & $2,9940.5 \%$ & $\mathrm{~m}$ \\
\hline Eric Dumazet & $2,9880.5 \%$ & $\mathrm{~m}$ \\
\hline Joe Perches & $2,9370.5 \%$ & $\mathrm{~m}$ \\
\hline Alex Deucher & $2,7570.4 \%$ & $\mathrm{~m}$ \\
\hline Daniel Vetter & $2,6880.4 \%$ & $\mathrm{~m}$ \\
\hline Laurent Pinchart & $2,6870.4 \%$ & $\mathrm{~m}$ \\
\hline Axel Lin & $2,6700.4 \%$ & $\mathrm{~m}$ \\
\hline Trond Myklebust & $2,5540.4 \%$ & $\mathrm{~m}$ \\
\hline Ben Skeggs & $2,5160.4 \%$ & $\mathrm{~m}$ \\
\hline Arnaldo Carvalho de Melo & $2,4560.4 \%$ & $\mathrm{~m}$ \\
\hline Bartlomiej Zolnierkiewicz & $2,3310.4 \%$ & $\mathrm{~m}$ \\
\hline Kuninori Morimoto & $2,3000.4 \%$ & $\mathrm{~m}$ \\
\hline Linus Walleij & $2,2810.4 \%$ & \\
\hline & & \\
\hline
\end{tabular}

Source (except gender) : "Top 30 kernel developers, 2.6.11-4.13" Linux Foundation 2017

Table 4 - Aggregated results

\begin{tabular}{|l|l|l|}
\hline Gender & $\mathrm{n}$ & Percentage \\
\hline Female & 0 & $0 \%$ \\
\hline Male & 30 & $100 \%$ \\
\hline
\end{tabular}

Table 4: Diversity among top 30 Linux kernel developers (2005-17) 


\section{References}

- "Linux kernel source tree". https://github.com/torvalds/linux/graphs/contributors ("Contributions to master, excluding merge commits")

- Linux Foundation. 2017. "Linux kernel development report". 